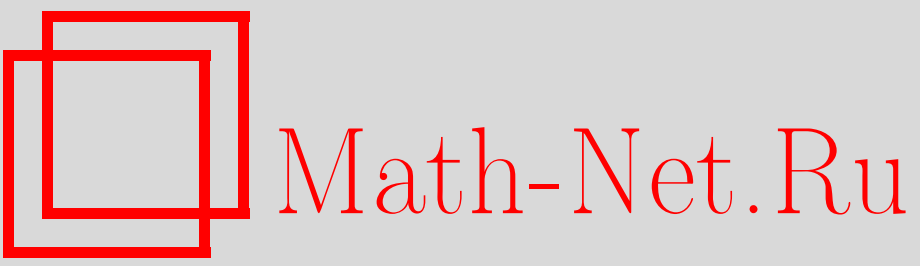

А. А. Джалилов, Х. К. Каршибоев, Предельные теоремы для времени попаданий отображений окружности с одной точкой излома, УМH, 2004, том 59, выпуск 1, 185-186

DOI: https://doi.org/10.4213/rm711

Использование Общероссийского математического портала Math-Net.Ru подразумевает, что вы прочитали и согласны с пользовательским соглашением

http://www . mathnet.ru/rus/agreement

Параметры загрузки:

IP: 54.210 .77 .194

26 апреля 2023 г., 15:56:55 


\title{
ПРЕДЕЛЬНЫЕ ТЕОРЕМЫ ДЛЯ ВРЕМЕНИ ПОПАДАНИЙ ОТОБРАЖЕНИЙ ОКРУЖНОСТИ С ОДНОЙ ТОЧКОЙ ИЗЛОМА
}

\author{
А. А. ДЖАлИЛОВ, Х. К. КАРшИБОЕВ
}

В настоящей работе изучаются гомеоморфизмы окружности, задаваемые функциями с одной точкой излома, в которой происходит скачок первой производной. Пусть $f(x)$ - непрерывная строго монотонная функция такая, что

a) $f(x+1)=f(x)+1$ для любого $x \in \mathbb{R}^{1}$;

b) существует точка $x_{c} \in S^{1}=[0,1)$ такая, что $f(x) \in C^{2+\varepsilon}\left(\left[x_{c}, x_{c}+1\right]\right)$ при некотором $\varepsilon>0, f^{\prime}(x)>$ const $>0$ для всех $x \in\left(x_{c}, x_{c}+1\right)$;

c) существуют конечные положительные односторонние производные $f_{-}^{\prime}\left(x_{c}\right), f_{+}^{\prime}\left(x_{c}\right)$ и $\sqrt{f^{\prime}\left(x_{c}-0\right) / f^{\prime}\left(x_{c}+0\right)}=c \neq 1$.

Определим гомеоморфизм окружности $T$, отвечающий $f: T x=\{f(x)\}, x \in S^{1}$, где $\{\cdot\}$ обозначает дробную часть числа. Пусть число вращения $\rho=\rho(T)$ иррационально и его разложение в непрерьвную дробь имеет вид: $\rho=\left[k_{1}, k_{2}, \ldots, k_{n}, \ldots\right]$. Обозначим через $\frac{p_{n}}{q_{n}}=$ $\left[k_{1}, k_{2}, \ldots, k_{n}\right], n \geqslant 1$, конечные дроби числа $\rho$. Числа $q_{n}$ удовлетворяют следующим рекуррентным соотношениям: $q_{n+1}=k_{n+1} q_{n}+q_{n-1}, q_{0}=1, q_{1}=k_{1}$. Обозначим $x_{0}=x_{c}$ и $x_{i}=T^{i} x_{0}, i \geqslant 1$, где $T^{i}$ обозначает $i$-ю итерацию $T$. Обозначим через $\Delta_{0}^{(n)}$ замкнутый отрезок, соединяющий точки $x_{0}$ и $x_{q_{n}}$. Заметим, что при нечетном $n$ точка $x_{q_{n}}$ лежит слева от $x_{0}$, а при чётном $n$ - справа. Положим $\Delta_{i}^{(n)}=T^{i} \Delta_{0}^{(n)}, i \geqslant 1$. Системы интервалов $\Delta_{i}^{(n-1)}, 0 \leqslant i<q n$, и $\Delta_{j}^{(n)}, 0 \leqslant j<q_{n-1}$, попарно не пересекаются (за исключением концевых точек) и образуют разбиение окружности (см. [1]). Полученное разбиение обозначим через $\xi_{n}$. Опишем процесс перехода от $\xi_{n-1}$ к $\xi_{n}$. Все отрезки $\Delta_{j}^{(n)}, 0 \leqslant j<q_{n-1}$, сохраняются, а каждой отрезок $\Delta_{i}^{(n-1)}$, $0 \leqslant i<q_{n}$, разбивается на $k_{n+1}+1$ отрезков: $\Delta_{i}^{(n-1)}=\Delta_{i}^{(n+1)} \cup \bigcup_{s=0}^{k_{n+1}-1} \Delta_{i+q_{n-1}+s q_{n}}^{(n)}$.

ЛЕмма 1. Пусть определяющая функция $f(x)$ гомеоморфизма Т удовлетворяет условиям (a)-(c) и число вращения $\rho$ иррачионально. Тогда для любого $\Delta^{(n)} \in \xi_{n}$ справедливо неравенство $\left|\Delta^{(n)}\right| \leqslant \mathrm{const} \cdot \lambda^{n}$, где $|\cdot|$ обозначает длину отрезка, а константа $\lambda \in(0,1)$.

Обозначим через $V_{n}$ отрезок, соединяющий точки $x_{q_{n-1}}$ и $x_{q_{n}}$. Обозначим через $N_{n}^{(k)}$ момент $k$-го попадания точки $x$ в $V_{n}$. Для каждого $k \geqslant 1$ определим $D_{n}^{(k)}(x)=N_{n}^{(k+1)}(x)-N_{n}^{(k)}(x)$, $n \geqslant 1, N_{n}^{(0)}(x) \equiv 0$. Заметим, что $N_{n}^{(1)}(x)$ принимает значения от 1 до $q_{n}$, а $D_{n}^{(k)}(x), k>1$, принимает всего два значения: $q_{n-1}$ и $q_{n}$. Определим следующие нормированные случайные величины: $\bar{D}_{n}^{(k)}(x)=q_{n}^{-1} D_{n}^{(k)}(x), k \geqslant 1$.

Посколку число вращения $\rho$ иррационально, отображение $T$ обладает единственной нормированной инвариантной мерой $\mu$. Обозначим через $\lambda$ меру Лебега на окружности. Обозначим $A_{n}^{(k)}(t)=\left\{x \in S^{1}: \bar{D}_{n}^{(k)}(x) \leqslant t\right\}$. Рассмотрим функции распределения случайных величин $\bar{D}_{n}^{(k)}(x)$ относительно мер $\mu$ и $\lambda$, т.е. $F_{n}^{(k)}(t)=\mu\left(A_{n}^{(k)}(t)\right), \Phi_{n}^{(k)}(t)=\lambda\left(A_{n}^{(k)}(t)\right), \forall t \in \mathbb{R}^{1}$.

Заметим, что функции $F_{n}^{(k)}(t)$ совпадают с соответствующими функциями распределения для линейного поворота $T_{\rho} x=\{x+\rho\}, x \in S^{1}$. В работе [2] доказано, что предельное распределение сходящейся подпоследователности $\left\{F_{n_{s}}^{(1)}(t)\right\}$, в зависимости от числа вращения $\rho$, является или равномерньм распределением, или непрерьвным кусочно-линейным распределением на $[0,1]$. При $k>1$ предел подпоследовательности $\left\{F_{n_{s}}^{(k)}(t)\right\}$ является или распределением для случайной величины $X \equiv 1$, или ступенчатым распределением с двумя точками разрьва.

Если диффеоморфизм гладко сопряжен с линейным поворотом $T_{\rho}$, то для последователшности $\left\{\Phi_{n}^{(k)}(t)\right\}$ все приведенные вьше утверждения, относящиеся к $\left\{F_{n}^{(k)}(t)\right\}$, также справедливы [2]. 
Заметим, что для гомеоморфизмов окружности с одной точкой излома и с иррациональньм числом вращения сопряжение является сингулярным [3].

Теорема 1. Предположим, что определяющая функиия $f(x)$ гомеоморфизма $T$ удовлетворяет условиям а)-c), а число вращения $\rho$ иррационально и его коэффициенты разложсения в непрерывную дробь ограничены. Пусть подпоследовательность функчий распределения $\left\{\Phi_{n_{s}}^{(1)}(t)\right\}$ сходится поточечно на $\mathbb{R}^{1} \kappa$ функиии распределения $\Phi^{(1)}(t)$. Тогда предельная функция $\Phi^{(1)}(t)$ непрерьвна на $\mathbb{R}^{1}$ и сингулярна на $[0,1]$, т.е. $\frac{d}{d t} \Phi^{(1)}(t)=0$ почти всюду.

Теорема 2. Предположим, что определяющая функция $f(x)$ гомеоморфизма $T$ и число вращения $\rho$ удовлетворяют условиям теоремы 1 . Пусть $k>1 u \lim _{s \rightarrow \infty} \Phi_{n_{s}}^{(k)}(t)=$ $\Phi^{(k)}(t), \forall t \in \mathbb{R}^{1}$. Тогда функция распределения $\Phi^{(k)}(t)$ является ступенчатой функцией с двумя точками разрыва из $(0,1)$.

Теперь приведем примеры гомеоморфизмов окружности, для которых последовательность $\left\{\Phi_{n}^{(k)}(t)\right\}_{n=1}^{\infty}$ прикаждом $k \geqslant 1$ сходится. Рассмотрим множество $X$, состоящее из пар непрерьвных строго возрастающих функций $(f(x), x \in[-1,0] ; g(x), x \in[0, \alpha])$, удовлетворяющих следующим условиям: 1) $f(0)=\alpha, \alpha>0, g(0)=-1, f(\alpha)=g(1) ; 2) f(-1)<0, \ldots, f^{(m)}(-1)<0$, $f^{(m+1)}(-1)>0$, где $f^{(k)}$ обозначает $k$-ю итерацию функции $f(x)$. Используя пару $(f, g)$ из $X$, можно построить гомеоморфизм окружности: $T x=f(x)$, если $x \in[-1,0)$, и $T x=g(x)$, если $x \in[0, \alpha)$. Обозначим $\rho_{m}=[m+1, m, \ldots, m, \ldots], m \geqslant 1$. Обозначим через $X_{m}$ подмножество $X$, состоящее из таких пар $(f, g)$, что число вращения соответствующего гомеоморфизма равно иррациональному числу $\rho_{m}$. На $X_{m}$ определим ренормгрупповое преобразование:

$$
R_{m}(f(x),-1 \leqslant x<0 ; g(x), 0 \leqslant x<\alpha)=(\widetilde{f}(x),-1 \leqslant x<0 ; \widetilde{g}(x), 0 \leqslant x<\widetilde{\alpha}),
$$

где $\widetilde{f}(x)=-\alpha^{-1} f^{m}(g(-\alpha x)), \widetilde{g}(x)=-\alpha^{-1} f(-\alpha x), \widetilde{\alpha}=-\alpha^{-1} f^{k}(-1)$. Положим $c=\frac{f_{-}^{\prime}(0)}{g_{+}^{\prime}(0)}$. $\frac{g_{-}^{\prime}(\alpha)}{f_{+}^{\prime}(-1)}$. В [4] доказано, что при фиксированном $c, c \neq 1$, преобразование $R_{m}$ имеет единственную периодическую траекторию $\left\{\left(f_{i}, g_{i}\right), i=1,2\right\}$ периода 2 . Функции $f_{i}(x), g_{i}(x), i=1,2$, являются дробно-линейньми. Обозначим через $T_{1}$ гомеоморфизм окружности $\left[-1, \alpha_{1}\right)$, соответствующий паре $\left(f_{1}, g_{1}\right)$ (пара $\left(f_{2}, g_{2}\right)$ изучается аналогично). Множество всех гомеоморфизмов, $C^{1}$-сопряженных с $T_{1}$, обозначим $B\left(T_{1}\right)$. Учитьвая, что $\left(f_{1}, g_{1}\right)$ является периодической точкой $R_{1}$, можно построить термодинамический формализм для всех гомеоморфизмов множества $B\left(T_{1}\right)[5]$.

Теорема 3. Пусть $T \in B\left(T_{1}\right)$. Тогда соответствующая последовательность функций распределения $\left\{\Phi_{n}^{(k)}(t)\right\}$ при каждом $k \geqslant 1$ сходится поточечно на $\mathbb{R}^{1} \kappa$ функции распределения $\Phi^{(k)}(t)$.

Авторы признательны Я. Г. Синаю за полезные обсуждения.

\section{СПИСОК ЛИТЕРАТУРЫ}

[1] Я.Г. Синай. Современные проблемы эргодической теории. М.: Физматлит, 1995. [2] Z. Coelho, E. de Faria // Israel J. Math. 1996. V. 93. P. 93-112. [3] А. А. Джалилов, К. М. Ханин // Функц. анализ и его прил. 1998. Т. 32. №3. С. 11-21. [4] К. M. Khanin, E. B. Vul // Adv. Soviet Math. 1991. V. 3. P. 57-98. [5] А. А. Джалилов // ТМф. 2003. T. 134. № 2. C. 190-206. 